\title{
ESCAP CovCAP survey of heads of academic departments to assess the perceived initial (April/May 2020) impact of the COVID-19 pandemic on child and adolescent psychiatry services
}

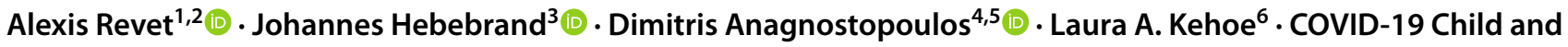 \\ Adolescent Psychiatry Consortium • Paul Klauser ${ }^{7,8}$
}

Received: 19 August 2020 / Accepted: 25 November 2020 / Published online: 20 January 2021

(c) Springer-Verlag GmbH Germany, part of Springer Nature 2021, corrected publication 2021

\begin{abstract}
In April 2020, the European Society for Child and Adolescent Psychiatry (ESCAP) Research Academy and the ESCAP Board launched the first of three scheduled surveys to evaluate the impact of the coronavirus disease 2019 (COVID-19) pandemic on child and adolescent psychiatry (CAP) services in Europe and to assess the abilities of CAP centers to meet the new challenges brought on by the crisis. The survey was a self-report questionnaire, using a multistage process, which was sent to 168 heads of academic CAP services in 24 European countries. Eighty-two responses ( 56 complete) from 20 countries, representing the subjective judgement of heads of CAP centers, were received between mid-April and mid-May 2020. Most respondents judged the impact of the crisis on the mental health of their patients as medium (52\%) or strong (33\%). A large majority of CAP services reported no COVID-19 positive cases among their inpatients and most respondents declared no or limited sick leaves in their team due to COVID-19. Outpatient, daycare, and inpatient units experienced closures or reductions in the number of treated patients throughout Europe. In addition, a lower referral rate was observed in most countries. Respondents considered that they were well equipped to handle COVID-19 patients despite a lack of protective equipment. Telemedicine was adopted by almost every team despite its sparse use prior to the crisis. Overall, these first results were surprisingly homogeneous, showing a substantially reduced patient load and a moderate effect of the COVID-19 crisis on psychopathology. The effect on the organization of CAP services appears profound. COVID-19 crisis has accelerated the adoption of new technologies, including telepsychiatry.
\end{abstract}

Keywords COVID-19 · Child and adolescent psychiatry · Telepsychiatry · Europe

\section{Introduction}

The coronavirus disease 2019 (COVID-19) pandemic is having a strong impact on the mental health of children and adolescents, their parents and families, and whole communities $[1,2]$. The fear of the disease, as well as the lockdown measures put in place by governments such as social distancing, quarantines, school closures, and intense hygiene measures, have raised concerns that existing mental health problems may worsen [3], or trigger persons to experience

The members of the COVID-19 Child and Adolescent Psychiatry Consortium group are mentioned in "Acknowldgements" section.

Alexis Revet

revet.a@chu-toulouse.fr

Extended author information available on the last page of the article new mental health problems [4]. In this context, child and adolescent psychiatry (CAP) services have a key role to play during the pandemic and thereafter to mitigate the effects of the crisis on the mental health of children and youth.

In April 2020, the European Society for Child and Adolescent Psychiatry (ESCAP) Research Academy, a network of young European researchers in CAP [5], and the ESCAP Board launched a survey to estimate the impact of COVID19 on CAP services in Europe and to assess the capability of CAP centers to meet the new challenges brought on by the crisis. University heads of CAP services in ESCAP membership countries were contacted by email and invited to complete an online survey. Cross-sectional surveys are re-scheduled for the end of the year 2020 and spring 2021. 


\section{Methods}

The survey is a self-report questionnaire, using a multi-stage process based on a Delphi approach [6]. JH, PK, AR, and LK first drew up a list of 60 items related to the topic. The items were ranked by importance then reduced and assembled in a final 38-item questionnaire, which was revised and approved by members of the ESCAP Board. It included items related to the following sections: current situation of the respondent's country or region; screening for COVID-19 in CAP service; COVID-19-induced changes in psychopathology; provision of CAP services; effects on teams, size and capacity of CAP services and current use of CAP services. A last section of the questionnaire allowed for free comments.

The REDCap® web application was used to securely diffuse an email inviting to fill out an online questionnaire and to anonymously collect the data. Email addresses from heads of CAP services working in university hospitals were provided by members of the ESCAP Board and the ESCAP Research Academy network. For this first step of the CovCAP survey, we did not receive contact details for the following eleven ESCAP member countries in due time: Bosnia \& Herzegovina, Croatia, Finland, Iceland, Israel, Lithuania, Portugal, Romania, Russia, United Kingdom, and Ukraine. Questionnaires were completed between April 16th and May 19th, 2020; the respondents were asked if they wanted to be included in the COVID-19 Child and Adolescent Psychiatry Consortium (see Table 1 and author list). Survey response data were analyzed using simple frequency statistics. Due to missing items, we provide the total number of respondents for the respective item in relevant paragraphs of the main text. The comments of 19 participants were assessed using thematic analysis to identify, analyze, and report patterns (i.e., themes) within data [7]. We systematically coded interesting features in the data and collated them into potential themes which were reported in the manuscript by specifying the number of occurrences. To protect anonymity, findings per country are presented only if at least two CAP services provided information. The data were pooled for the five countries with only a single informant (category "Others" in Tables 1 and 2).

\section{Results}

\section{Characteristics of respondents}

168 heads of CAP university services in 24 countries were contacted by email and 82 responded providing an overall response rate of $49 \%$ (Table 1). Among all questionnaires returned, 56 were filled in completely; 19 included free comments. Twenty countries were represented (Tables 1 and 2), among which Germany had the highest number of responses ( $n=18 / 82$ respondents; $22 \%$ ). A single response only was obtained from The Netherlands, Estonia, Bulgaria, Serbia, and Slovakia. We did not get any replies from Albania, Cyprus, Norway, and Sweden.

\section{Lockdown measures and school closures by country}

All respondents reported lockdown measures in their countries, mostly rated as strict $(n=48 / 80 ; 60 \%)$. Schools had been closed "fully" or "partially" (combined $n=79 / 81 ; 98 \%$ ) for a mean of almost 6 weeks (mean $=5.8$ weeks; standard deviation; SD: 1.5) upon submission of the questionnaire.

\section{COVID-19 infections among patients and therapeutic teams}

The vast majority of CAP services reported no history of COVID-19 positive cases among their inpatients $(n=52 / 63$; $83 \%)$. Most respondents $(n=44 / 57 ; 77 \%)$ declared no or very limited $(<1 \%$ of the team) current sick leave in their team due to COVID-19. Twenty-four (out of $58 ; 41 \%$ )

Table 1 Number of head of child and adolescent psychiatry services contacted and number of answers per country

\begin{tabular}{llllllllllllllllll}
\hline Nationalities of respondents & AT & BE & CZ & DK & FR & DE & GR & HU & IE & IT & PL & SI & ES & CH & TR & Others & Total \\
\hline Number of head of CAP services contacted & 8 & 9 & 5 & 12 & 10 & 33 & 11 & 10 & 16 & 2 & 5 & 3 & 10 & 8 & 9 & 17 & 168 \\
Number of respondents & 2 & 2 & 2 & 5 & 5 & 18 & 6 & 7 & 4 & 2 & 2 & 2 & 5 & 6 & 9 & 5 & 82 \\
Number of complete questionnaires & 2 & 1 & 1 & 3 & 4 & 14 & 5 & 4 & 3 & 2 & 2 & 2 & 2 & 3 & 3 & 5 & 56 \\
\hline
\end{tabular}

International Organization for Standardization (ISO) countries codes: $A T$ Austria, BE Belgium, $C Z$ Czech Republic, $D K$ Denmark, $F R$ France, $D E$ Germany, GR Greece, HU Hungary, IE Ireland, IT Italy, PL Poland, SI Slovenia, ES Spain, CH Switzerland, TR Turkey

$C A P$ child, and adolescent psychiatry, $S D$ standard deviation

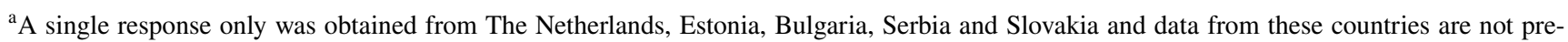
sented individually to protect anonymity of answers (i.e. number of questionnaires filled $<2$ )

We were, unfortunately, unable to obtain contact information in time for this first step of the CovCAP survey for eleven countries (Bosnia \& Herzegovina, Croatia, Finland, Iceland, Israel, Lithuania, Portugal, Romania, Russia, United Kingdom and Ukraine). We did not receive replies from Albania, Cyprus, Norway and Sweden 
Table 2 Main answers of respondents to the initial COVID-19 questionnaire (see the respective item in first column for the number of available responses)

\begin{tabular}{|c|c|c|c|c|c|c|c|c|c|c|c|c|c|c|c|c|c|}
\hline & AT & BE & $\mathrm{CZ}$ & DK & FR & DE & GR & HU & IE & IT & PL & SI & ES & CH & TR & Others $^{1}$ & Total \\
\hline \multicolumn{18}{|c|}{ Incidence of COVID-19 and reported lockdown measures and school closures by country } \\
\hline $\begin{array}{l}\text { Incidence COVID-19/100,000 } \\
(30.04 .2020)^{2}\end{array}$ & 171.6 & 418.6 & 71.7 & 161.5 & 256.3 & 194.6 & 24.9 & 28.7 & 417.4 & 339.8 & 34.0 & 68.7 & 456.5 & 341.9 & 142.5 & NA & \\
\hline \multicolumn{18}{|l|}{ Type of COVID-19 lockdown $(n=80)$} \\
\hline None & 0 & 0 & 0 & 0 & 0 & 0 & 0 & 0 & 0 & 0 & 0 & 0 & 0 & 0 & 0 & 0 & 0 \\
\hline Loose & 0 & 0 & 0 & 2 & 0 & 6 & 0 & 3 & 0 & 0 & 0 & 0 & 0 & 3 & 2 & 2 & $18(22.5 \%)$ \\
\hline Strict & 2 & 2 & 2 & 3 & 4 & 9 & 3 & 4 & 3 & 0 & 2 & 0 & 3 & 2 & 7 & 2 & $48(60 \%)$ \\
\hline Very strict & 0 & 0 & 0 & 0 & 1 & 1 & 3 & 0 & 1 & 2 & 0 & 2 & 2 & 1 & 0 & 1 & $14(17.5 \%)$ \\
\hline \multicolumn{18}{|l|}{ School closure $(n=81)$} \\
\hline & 0 & 0 & 0 & 0 & 0 & 0 & 0 & 1 & 0 & 0 & 0 & 0 & 0 & 0 & 0 & 1 & $2(2.5 \%)$ \\
\hline Partially & 0 & 0 & 0 & 4 & 0 & 2 & 0 & 0 & 0 & 0 & 0 & 0 & 0 & 0 & 0 & 0 & $6(7.4 \%)$ \\
\hline Yes & 2 & 2 & 2 & 1 & 5 & 15 & 6 & 6 & 4 & 2 & 2 & 2 & 5 & 6 & 9 & 4 & $73(90.1 \%)$ \\
\hline $\begin{array}{l}\text { Number of weeks of school closure } \\
(n=76 ; \text { mean }(S D))\end{array}$ & $\begin{array}{l}5.5 \\
(2.1) \\
\end{array}$ & $\begin{array}{l}5 \\
(0) \\
\end{array}$ & $\begin{array}{l}7.3 \\
(1.1) \\
\end{array}$ & $\begin{array}{l}5.3 \\
(1.9)\end{array}$ & $\begin{array}{l}5 \\
(1.9) \\
\end{array}$ & $\begin{array}{l}5.8 \\
(1.3)\end{array}$ & $\begin{array}{l}5.5 \\
(1)\end{array}$ & $\begin{array}{l}5.7 \\
(2.3)\end{array}$ & $\begin{array}{l}6 \\
(2.2) \\
\end{array}$ & $\begin{array}{l}4.5 \\
(0.7) \\
\end{array}$ & $\begin{array}{l}4.5 \\
(0.7)\end{array}$ & $\begin{array}{l}5 \\
(0)\end{array}$ & $\begin{array}{l}6.6 \\
(1.1) \\
\end{array}$ & $\begin{array}{l}5.2 \\
(0.8) \\
\end{array}$ & $\begin{array}{l}7.2 \\
(1.3)\end{array}$ & $\begin{array}{l}6 \\
(1.4) \\
\end{array}$ & $\begin{array}{l}5.8 \\
(1.5)\end{array}$ \\
\hline \multicolumn{18}{|c|}{ Reported COVID-19 infections among patients and therapeutic teams } \\
\hline \multicolumn{18}{|l|}{$\begin{array}{l}\text { Please indicate the total number of } \\
\text { confirmed (tested positive) COVID-19 } \\
\text { inpatients you have had in your CAP } \\
\text { service to date. }(n=63)\end{array}$} \\
\hline None & 2 & 1 & 1 & 2 & 3 & 15 & 6 & 5 & 3 & 1 & 1 & 2 & 1 & 2 & 2 & 5 & $52(82.5 \%)$ \\
\hline 1 & 0 & 0 & 0 & 0 & 0 & 1 & 0 & 0 & 0 & 1 & 1 & 0 & 2 & 1 & 0 & 0 & $6(9.5 \%)$ \\
\hline$>1$ & 0 & 0 & 0 & 1 & 2 & 0 & 0 & 1 & 0 & 0 & 0 & 0 & 1 & 0 & 0 & 0 & $5(7.9 \%)$ \\
\hline \multicolumn{18}{|l|}{$\begin{array}{l}\text { Has a patient and/or staff member } \\
\text { contracted COVID-19 from a positive } \\
\text { patient in your clinic? (please answer } \\
\text { affirmatively even if transmission has } \\
\text { not been proven) (n=58) }\end{array}$} \\
\hline No & 2 & 1 & 1 & 3 & 3 & 15 & 5 & 4 & 3 & 1 & 2 & 2 & 0 & 2 & 3 & 5 & $52(89.7 \%)$ \\
\hline Single patient & 0 & 0 & 0 & 0 & 0 & 0 & 0 & 0 & 0 & 0 & 0 & 0 & 0 & 0 & 0 & 0 & 0 \\
\hline More than one patient & 0 & 0 & 0 & 0 & 0 & 0 & 0 & 0 & 0 & 0 & 0 & 0 & 0 & 0 & 0 & 0 & 0 \\
\hline Single staff member & 0 & 0 & 0 & 0 & 0 & 0 & 0 & 0 & 0 & 0 & 0 & 0 & 1 & 1 & 0 & 0 & $2(3.4 \%)$ \\
\hline More than one staff member & 0 & 0 & 0 & 0 & 2 & 0 & 0 & 0 & 0 & 1 & 0 & 0 & 1 & 0 & 0 & 0 & $4(6.9 \%)$ \\
\hline \multicolumn{18}{|l|}{$\begin{array}{l}\text { How many of your staff members have } \\
\text { been infected (tested positive) with } \\
\text { COVID-19 to date? }(n=58)\end{array}$} \\
\hline Not aware of a single case & 2 & 0 & 0 & 1 & 0 & 10 & 5 & 2 & 3 & 1 & 2 & 1 & 1 & 0 & 3 & 3 & $34(58.6 \%)$ \\
\hline$<1 \%$ & 0 & 1 & 1 & 2 & 0 & 2 & 0 & 2 & 0 & 0 & 0 & 1 & 0 & 2 & 0 & 0 & $11(19 \%)$ \\
\hline $1-5 \%$ & 0 & 0 & 0 & 0 & 3 & 2 & 0 & 0 & 0 & 0 & 0 & 0 & 1 & 1 & 0 & 2 & $9(15.5 \%)$ \\
\hline $5-10 \%$ & 0 & 0 & 0 & 0 & 2 & 1 & 0 & 0 & 0 & 1 & 0 & 0 & 0 & 0 & 0 & 0 & $4(6.9 \%)$ \\
\hline $10-25 \%$ & 0 & 0 & 0 & 0 & 0 & 0 & 0 & 0 & 0 & 0 & 0 & 0 & 0 & 0 & 0 & 0 & 0 \\
\hline$>50 \%$ & 0 & 0 & 0 & 0 & 0 & 0 & 0 & 0 & 0 & 0 & 0 & 0 & 0 & 0 & 0 & 0 & 0 \\
\hline \multicolumn{18}{|l|}{$\begin{array}{l}\text { If applicable, how many staff have } \\
\text { died due to the COVID-19? }(n=40)\end{array}$} \\
\hline 0 & 2 & 1 & 1 & 2 & 4 & 11 & 1 & 3 & 2 & 1 & 0 & 2 & 1 & 3 & 2 & 3 & $39(97.5 \%)$ \\
\hline 1 & 0 & 0 & 0 & 1 & 0 & 0 & 0 & 0 & 0 & 0 & 0 & 0 & 0 & 0 & 0 & 0 & $1(2.5 \%)$ \\
\hline \multicolumn{18}{|l|}{$\begin{array}{l}\text { Currently (today), how many numbers } \\
\text { of staff are on sick leave, most likely } \\
\text { due to Covid-19? }(n=57))\end{array}$} \\
\hline Not aware of a single case & 2 & 0 & 0 & 0 & 0 & 7 & 5 & 3 & 1 & 0 & 2 & 1 & 1 & 1 & 3 & 3 & $29(50.9 \%)$ \\
\hline$<1 \%$ & 0 & 0 & 1 & 3 & 3 & 4 & 0 & 1 & 0 & 1 & 0 & 1 & 1 & 0 & 0 & 0 & $15(26.3 \%)$ \\
\hline $1-5 \%$ & 0 & 1 & 0 & 0 & 2 & 2 & 0 & 0 & 0 & 0 & 0 & 0 & 0 & 1 & 0 & 2 & $8(14 \%)$ \\
\hline $5-10 \%$ & 0 & 0 & 0 & 0 & 0 & 2 & 0 & 0 & 1 & 0 & 0 & 0 & 0 & 0 & 0 & 0 & $3(5.3 \%)$ \\
\hline $10-25 \%$ & 0 & 0 & 0 & 0 & 0 & 0 & 0 & 0 & 0 & 1 & 0 & 0 & 0 & 1 & 0 & 0 & $2(3.5 \%)$ \\
\hline$>50 \%$ & 0 & 0 & 0 & 0 & 0 & 0 & 0 & 0 & 0 & 0 & 0 & 0 & 0 & 0 & 0 & 0 & 0 \\
\hline \multicolumn{18}{|l|}{$\begin{array}{l}\text { Have you had any staff that have been } \\
\text { re-assigned to critical care? }(n=57)\end{array}$} \\
\hline Yes & 0 & 0 & 0 & 2 & 3 & 4 & 0 & 1 & 0 & 0 & 0 & 1 & 0 & 0 & 1 & 0 & $12(21.1 \%)$ \\
\hline No & 2 & 1 & 1 & 1 & 2 & 11 & 5 & 3 & 2 & 2 & 2 & 1 & 2 & 3 & 2 & 5 & $45(78.9 \%)$ \\
\hline \multicolumn{18}{|c|}{ Screening for COVID-19 in CAP services } \\
\hline $\begin{array}{l}\text { When admitting an inpatient, how do } \\
\text { you screen for COVID-19? }(n=61)\end{array}$ & & & & & & & & & & & & & & & & & \\
\hline $\begin{array}{l}\text { As usual (reference 2019, no } \\
\text { screening for COVID-19 infection) }\end{array}$ & 0 & 0 & 0 & 0 & 0 & 0 & 1 & 1 & 1 & 0 & 0 & 0 & 0 & 0 & 0 & 0 & $3(4.9 \%)$ \\
\hline $\begin{array}{l}\text { We screen for signs of infection in a } \\
\text { patient prior to admission }\end{array}$ & 0 & 0 & 0 & 1 & 0 & 2 & 2 & 0 & 0 & 0 & 2 & 0 & 3 & 0 & 0 & 1 & $11(18 \%)$ \\
\hline $\begin{array}{l}\text { We screen for signs of infection in a } \\
\text { patient and their household members } \\
\text { prior to admission }\end{array}$ & 2 & 1 & 0 & 1 & 2 & 9 & 1 & 4 & 1 & 1 & 0 & 0 & 2 & 2 & 2 & 1 & $29(47.5 \%)$ \\
\hline We require a negative COVID-19 test & 0 & 0 & 1 & 1 & 3 & 5 & 0 & 1 & 0 & 1 & 0 & 2 & 0 & 1 & 0 & 3 & $18(29.5 \%)$ \\
\hline $\begin{array}{l}\text { How rapidly can you obtain a } \\
\text { COVID-19 test result for an } \\
\text { inpatient? }(n=61)\end{array}$ & & & & & & & & & & & & & & & & & \\
\hline $\begin{array}{r}\text { We have no experience; no inpatient } \\
\text { has been tested by us or upon our } \\
\text { request }\end{array}$ & 0 & 0 & 0 & 0 & 0 & 0 & 3 & 1 & 1 & 0 & 0 & 0 & 1 & 0 & 1 & 0 & $7(11.5 \%)$ \\
\hline Within 24 hours & 2 & 0 & 1 & 3 & 2 & 11 & 1 & 4 & 1 & 2 & 0 & 2 & 2 & 2 & 0 & 4 & $37(60.7 \%)$ \\
\hline $24-48$ hours & 0 & 1 & 0 & 0 & 3 & 3 & 1 & 1 & 1 & 0 & 2 & 0 & 0 & 1 & 1 & 0 & $14(23 \%)$ \\
\hline 48-72 hours & 0 & 0 & 0 & 0 & 0 & 1 & 0 & 0 & 0 & 0 & 0 & 0 & 0 & 0 & 0 & 1 & $2(3.3 \%)$ \\
\hline 72 hours & 0 & 0 & 0 & 0 & 0 & 1 & 0 & 0 & 0 & 0 & 0 & 0 & 0 & 0 & 0 & 0 & $1(1.6 \%)$ \\
\hline
\end{tabular}


Table 2 (continued)

\begin{tabular}{|c|c|c|c|c|c|c|c|c|c|c|c|c|c|c|c|c|c|}
\hline \multirow{2}{*}{\multicolumn{18}{|c|}{ Impact on service provision of }} \\
\hline & & & & & & & & & & & & & & & & & \\
\hline Not at all & 0 & 0 & 0 & 0 & 0 & 0 & 0 & 0 & 0 & 0 & 0 & 0 & 0 & 1 & 1 & 0 & $2(3.3 \%)$ \\
\hline To a minor degree only & 0 & 1 & 0 & 1 & 0 & 6 & 1 & 0 & 0 & 0 & 0 & 0 & 0 & 0 & 1 & 0 & $10(16.9 \%)$ \\
\hline To a major degree & 2 & 0 & 1 & 2 & 3 & 8 & 3 & 5 & 3 & 1 & 2 & 2 & 1 & 1 & 1 & 5 & $40(67.8 \%)$ \\
\hline Extremely & 0 & 0 & 0 & 0 & 2 & 1 & 1 & 0 & 0 & 1 & 0 & 0 & 1 & 1 & 0 & 0 & $7(11.9 \%)$ \\
\hline \multicolumn{18}{|l|}{$\begin{array}{l}\text { How have the regulatory measures } \\
\text { placed in your country affected your } \\
\text { clinical services? ( } n=56)\end{array}$} \\
\hline Closure of day care units & 2 & 0 & 0 & 1 & 5 & 11 & 2 & 2 & 2 & 1 & 2 & 2 & 1 & 3 & 2 & 2 & $38(67.9 \%)$ \\
\hline $\begin{array}{r}\text { Closure of specific inpatient } \\
\text { wards/units }\end{array}$ & 2 & 0 & 0 & 0 & 2 & 5 & 1 & 2 & 1 & 0 & 1 & 1 & 0 & 1 & 1 & 1 & $18(32.1 \%)$ \\
\hline $\begin{array}{r}\text { Restricted access to our outpatient } \\
\text { unit (e.g. for medically relevant cases } \\
\text { or emergencies only) }\end{array}$ & 2 & 1 & 1 & 2 & 4 & 11 & 5 & 4 & 3 & 2 & 2 & 2 & 2 & 2 & 3 & 4 & $50(89.3 \%)$ \\
\hline \multicolumn{18}{|l|}{$\begin{array}{l}\text { Restrictions in place for parental } \\
\text { access to inpatients }(n=55)\end{array}$} \\
\hline Yes & 1 & 1 & 1 & 3 & 5 & 10 & 3 & 2 & 2 & 2 & 2 & 2 & 1 & 2 & 0 & 4 & $41(74.5 \%)$ \\
\hline No & 1 & 0 & 0 & 0 & 0 & 5 & 0 & 3 & 0 & 0 & 0 & 0 & 1 & 1 & 2 & 1 & $14(25.5 \%)$ \\
\hline \multicolumn{18}{|l|}{$\begin{array}{l}\text { Have you changed policies with } \\
\text { respect to contacts between patients } \\
\text { and caregivers? }(n=57)\end{array}$} \\
\hline No & 0 & 0 & 0 & 0 & 1 & 0 & 0 & 1 & 0 & 0 & 0 & 0 & 0 & 1 & 0 & 1 & $4(7 \%)$ \\
\hline Slight restrictions & 0 & 1 & 0 & 1 & 0 & 5 & 0 & 0 & 0 & 1 & 0 & 0 & 0 & 0 & 0 & 1 & $9(15.8 \%)$ \\
\hline Moderate restrictions & 1 & 0 & 0 & 2 & 1 & 6 & 3 & 3 & 1 & 0 & 0 & 0 & 2 & 1 & 2 & 1 & $23(40.4 \%)$ \\
\hline Severe restrictions & 1 & 0 & 1 & 0 & 3 & 4 & 1 & 1 & 2 & 1 & 2 & 2 & 0 & 1 & 0 & 2 & $21(36.8 \%)$ \\
\hline \multicolumn{18}{|l|}{$\begin{array}{l}\text { Have any parts of your service been } \\
\text { closed or transformed for emergency } \\
\text { COVID-19 use? }(n=59)\end{array}$} \\
\hline 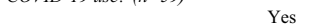 & 2 & 0 & 1 & 2 & 4 & 11 & 1 & 4 & 1 & 1 & 1 & 1 & 0 & 3 & 1 & 2 & $35(59.3 \%)$ \\
\hline No & 0 & 1 & 0 & 1 & 1 & 4 & 4 & 1 & 2 & 1 & 1 & 1 & 2 & 0 & 2 & 3 & $24(40.7 \%)$ \\
\hline $\begin{array}{l}\text { If you can, please indicate the average } \\
\text { daily number of inpatients (beds) } \\
\text { (pertains to estimated average number } \\
\text { of inpatients per day in } 2019 \\
\text { irrespective of initial referrals or } \\
\text { recontacts) at your hospital. }(n=44 ; \\
\text { mean (SD)) }\end{array}$ & $\begin{array}{l}29 \\
(5.7)\end{array}$ & - & $\begin{array}{l}15 \\
(-)\end{array}$ & $\begin{array}{l}34.7 \\
(31.0)\end{array}$ & $\begin{array}{l}33.3 \\
(23.6)\end{array}$ & $\begin{array}{l}50.8 \\
(19.6)\end{array}$ & $6(-)$ & $\begin{array}{l}42.5 \\
(10.6)\end{array}$ & $7(9.9)$ & $\begin{array}{l}11 \\
(5.7)\end{array}$ & $28(0)$ & $\begin{array}{l}17 \\
(9.9)\end{array}$ & $\begin{array}{l}15.5 \\
(21.9)\end{array}$ & $\begin{array}{l}35 \\
(28.3)\end{array}$ & $2(-)$ & $19.4(4.7)$ & $31.9(22)$ \\
\hline $\begin{array}{l}\text { Please provide today's numbers for } \\
\text { inpatients (number of beds occupied). } \\
\text { If you are filling the questionnaire } \\
\text { during the weekend, estimate the } \\
\text { average occupancy during the last } \\
\text { week. }(n=47 ; \text { mean }(S D))\end{array}$ & $\begin{array}{l}15 \\
(9.9)\end{array}$ & - & $9(-)$ & $\begin{array}{l}29 \\
(27.1)\end{array}$ & $\begin{array}{l}16 \\
(16.2)\end{array}$ & $\begin{array}{l}31.4 \\
(13.2)\end{array}$ & $2(-)$ & $\begin{array}{l}2.5 \\
(2.4)\end{array}$ & $7(9.9)$ & $\begin{array}{l}11.5 \\
(9.2)\end{array}$ & $\begin{array}{l}6.5 \\
(6.4)\end{array}$ & $\begin{array}{l}11 \\
(1.4)\end{array}$ & $\begin{array}{l}15.5 \\
(21.9)\end{array}$ & $\begin{array}{l}34 \\
(32.5)\end{array}$ & $0(-)$ & $8.2(4.8)$ & $18.1(16.6)$ \\
\hline $\begin{array}{l}\text { If you can, please indicate the average } \\
\text { daily number of outpatients (pertains } \\
\text { to estimated average number of } \\
\text { outpatients per day in } 2019 \\
\text { irrespective of initial referrals or } \\
\text { recontacts) at your hospital. }(n=46 ; \\
\text { mean (SD)) }\end{array}$ & $\begin{array}{l}14 \\
(8.5)\end{array}$ & - & $8(-)$ & $\begin{array}{l}200 \\
(-)\end{array}$ & $\begin{array}{l}81.3 \\
(45.0)\end{array}$ & $\begin{array}{l}45.9 \\
(31.1)\end{array}$ & $\begin{array}{l}19.6 \\
(12.2)\end{array}$ & $\begin{array}{l}50 \\
(14.1)\end{array}$ & $\begin{array}{l}13.5 \\
(9.2)\end{array}$ & $\begin{array}{l}35 \\
(7.1)\end{array}$ & $\begin{array}{l}10.5 \\
(6.4)\end{array}$ & $\begin{array}{l}20 \\
(14.1)\end{array}$ & $\begin{array}{l}82.5 \\
(95.5)\end{array}$ & $\begin{array}{l}172.5 \\
(109.6)\end{array}$ & $\begin{array}{l}66.7 \\
(41.6)\end{array}$ & $224(378.9)$ & $69.9(135.3)$ \\
\hline $\begin{array}{l}\text { Please provide today's numbers for } \\
\text { outpatients (outpatient visits). If you } \\
\text { are filling the questionnaire during } \\
\text { the weekend, estimate the average } \\
\text { occupancy during the last week. } \\
(n=48 ; \text { mean (SD)) }\end{array}$ & $\begin{array}{l}4 \\
(5.7)\end{array}$ & - & $3(-)$ & $\begin{array}{l}200 \\
(-)\end{array}$ & $\begin{array}{l}12.3 \\
(15.7)\end{array}$ & $\begin{array}{l}26 \\
(22.8)\end{array}$ & $\begin{array}{l}3 \\
(2.1)\end{array}$ & $\begin{array}{l}13.5 \\
(18.4)\end{array}$ & $10(14.1)$ & $\begin{array}{l}27.5 \\
(31.8)\end{array}$ & $\begin{array}{l}9.5 \\
(0.7)\end{array}$ & $\begin{array}{l}8.5 \\
(2.1)\end{array}$ & $\begin{array}{l}75 \\
(21.2)\end{array}$ & $\begin{array}{l}70 \\
(42.4)\end{array}$ & $\begin{array}{l}15.7 \\
(10.7)\end{array}$ & $23.8(8.5)$ & $24.7(35.2)$ \\
\hline $\begin{array}{l}\text { On a scale of } 0 \text { to } 100, \text { how well is } \\
\text { your team mentally equipped to } \\
\text { handle COVID-19 patients? }(n=57 \text {; } \\
\text { mean (SD)) }\end{array}$ & $\begin{array}{l}65 \\
(21.2)\end{array}$ & - & $\begin{array}{l}62 \\
(-)\end{array}$ & $\begin{array}{l}59 \\
(16.5)\end{array}$ & $\begin{array}{l}74 \\
(22.0)\end{array}$ & $\begin{array}{l}67.6 \\
(15.9)\end{array}$ & $\begin{array}{l}55.4 \\
(31.3)\end{array}$ & $\begin{array}{l}60 \\
(11.5)\end{array}$ & $\begin{array}{l}44.7 \\
(33.3)\end{array}$ & $\begin{array}{l}52 \\
(2.8)\end{array}$ & $\begin{array}{l}67.5 \\
(24.7)\end{array}$ & $\begin{array}{l}29 \\
(11.3)\end{array}$ & $\begin{array}{l}63 \\
(24.0)\end{array}$ & $\begin{array}{l}68 \\
(10.1)\end{array}$ & $\begin{array}{l}78.3 \\
(7.6)\end{array}$ & $60.4(11.7)$ & $\begin{array}{l}60.4 \\
(12.0)\end{array}$ \\
\hline \multicolumn{18}{|l|}{$\begin{array}{l}\text { What organization-directed } \\
\text { interventions has your service taken to } \\
\text { reduce the stress on your team } \\
\text { members? (n=59) }\end{array}$} \\
\hline None & 0 & 0 & 0 & 0 & 0 & 1 & 0 & 0 & 0 & 0 & 0 & 0 & 0 & 0 & 0 & 0 & $1(1.7 \%)$ \\
\hline $\begin{array}{r}\text { We allow for changes in the work } \\
\text { schedule }\end{array}$ & 2 & 1 & 1 & 1 & 4 & 13 & 4 & 4 & 1 & 1 & 2 & 1 & 2 & 2 & 3 & 5 & $47(79.7 \%)$ \\
\hline $\begin{array}{l}\text { We allow parents to stay at home if } \\
\text { their children are unable to attend } \\
\text { school }\end{array}$ & 2 & 0 & 1 & 1 & 2 & 10 & 5 & 2 & 1 & 0 & 1 & 2 & 1 & 1 & 2 & 4 & $35(59.3 \%)$ \\
\hline $\begin{array}{r}\text { Extra childcare (which also accept } \\
\text { schoolers) organized by your } \\
\text { institution }\end{array}$ & 1 & 1 & 0 & 1 & 3 & 6 & 0 & 1 & 0 & 0 & 0 & 1 & 0 & 1 & 0 & 1 & $16(27.1 \%)$ \\
\hline $\begin{array}{r}\text { We encourage home office but do not } \\
\text { allow patient contacts }\end{array}$ & 1 & 0 & 1 & 0 & 2 & 5 & 3 & 2 & 1 & 0 & 1 & 0 & 0 & 2 & 1 & 3 & $22(37.3 \%)$ \\
\hline $\begin{array}{r}\text { We encourage the use of home-based } \\
\text { calls/video conferences to } \\
\text { counsel/treat patients and families }\end{array}$ & 2 & 0 & 0 & 1 & 5 & 7 & 3 & 3 & 2 & 2 & 1 & 0 & 2 & 3 & 2 & 2 & $35(59.3 \%)$ \\
\hline
\end{tabular}


Table 2 (continued)

\begin{tabular}{|c|c|c|c|c|c|c|c|c|c|c|c|c|c|c|c|c|c|}
\hline $\begin{array}{l}\text { We have measures in place to improve } \\
\text { team-work }\end{array}$ & 0 & 1 & 0 & 1 & 3 & 3 & 0 & 2 & 2 & 1 & 0 & 0 & 1 & 1 & 1 & 1 & $17(28.8 \%)$ \\
\hline $\begin{array}{l}\text { Supervisions } \\
\end{array}$ & 2 & 1 & 0 & 0 & 4 & 4 & 1 & 1 & 0 & 1 & 0 & 1 & 1 & 1 & 3 & 2 & $22(37.3 \%)$ \\
\hline Mental health training program & 0 & 0 & 0 & 0 & 3 & 1 & 1 & 2 & 0 & 0 & 0 & 0 & 0 & 0 & 2 & 1 & $10(16.9 \%)$ \\
\hline Other & 0 & 0 & 0 & 1 & 2 & 1 & 0 & 0 & 0 & 0 & 1 & 0 & 0 & 0 & 0 & 0 & $5(8.5 \%)$ \\
\hline $\begin{array}{l}\text { On a scale of } 0 \text { to } 100, \text { how well is the } \\
\text { infrastructure of your service } \\
\text { equipped to handle COVID-19 } \\
\text { patients? ( } n=56 ; \text { mean (SD)) }\end{array}$ & $\begin{array}{l}59 \\
(36.8)\end{array}$ & - & $\begin{array}{l}66 \\
(-)\end{array}$ & $\begin{array}{l}68 \\
(7.5)\end{array}$ & $\begin{array}{l}77.6 \\
(20.5)\end{array}$ & $\begin{array}{l}59.1 \\
(22.0)\end{array}$ & $\begin{array}{l}40.2 \\
(30.7)\end{array}$ & $\begin{array}{l}53 \\
(26.8)\end{array}$ & $\begin{array}{l}27.7 \\
(29.3)\end{array}$ & $\begin{array}{l}39.5 \\
(3.5)\end{array}$ & $\begin{array}{l}60 \\
(14.1)\end{array}$ & $\begin{array}{l}40.5 \\
(14.8)\end{array}$ & $\begin{array}{l}82 \\
(-)\end{array}$ & $\begin{array}{l}68.3 \\
(24.6)\end{array}$ & $\begin{array}{l}76.7 \\
(5.8)\end{array}$ & $42.4(35.9)$ & $\begin{array}{l}57.2 \\
(19.6)\end{array}$ \\
\hline \multicolumn{18}{|l|}{$\begin{array}{l}\text { Does your team have appropriate } \\
\text { personal protective equipment (masks, } \\
\text { gloves, eye shields, etc.)? }(n=58)\end{array}$} \\
\hline No & 0 & 0 & 0 & 0 & 0 & 0 & 0 & 0 & 0 & 0 & 0 & 0 & 0 & 0 & 0 & 1 & $1(1.7 \%)$ \\
\hline Partially & 1 & 1 & 1 & 0 & 3 & 6 & 4 & 3 & 3 & 1 & 2 & 2 & 2 & 1 & 1 & 3 & $34(58.6 \%)$ \\
\hline Yes, fully protected & 1 & 0 & 0 & 3 & 2 & 9 & 1 & 1 & 0 & 1 & 0 & 0 & 0 & 2 & 2 & 1 & $23(39.7 \%)$ \\
\hline \multicolumn{18}{|l|}{$\begin{array}{l}\text { Have you established a crisis hotline } \\
\text { for families/adolescents with respect } \\
\text { to COVID-19 and its implications? } \\
\text { (e.g. social distancing/quarantine) } \\
\text { (n=61) }\end{array}$} \\
\hline Yes & 2 & 1 & 1 & 1 & 3 & 9 & 1 & 4 & 0 & 1 & 0 & 2 & 2 & 2 & 1 & 3 & $33(54.1 \%)$ \\
\hline No & 0 & 0 & 0 & 2 & 2 & 7 & 4 & 1 & 3 & 1 & 2 & 0 & 1 & 1 & 2 & 2 & $28(45.9 \%)$ \\
\hline \multicolumn{18}{|l|}{$\begin{array}{l}\text { Have your physicians/psychologists } \\
\text { adopted the use of telemedicine to } \\
\text { replace clinical appointments? }(n=60)\end{array}$} \\
\hline No & 0 & 0 & 0 & 0 & 0 & 0 & 0 & 0 & 1 & 0 & 0 & 0 & 0 & 0 & 1 & 1 & $3(5 \%)$ \\
\hline Yes & 2 & 1 & 1 & 3 & 4 & 16 & 5 & 5 & 2 & 2 & 2 & 2 & 3 & 3 & 2 & 4 & $57(95 \%)$ \\
\hline \multicolumn{18}{|l|}{$\begin{array}{l}\text { Was telemedicine already available in } \\
\text { your CAP service before the } \\
\text { pandemic? }(n=61)\end{array}$} \\
\hline No & 2 & 0 & 0 & 1 & 3 & 14 & 5 & 4 & 3 & 2 & 2 & 0 & 2 & 3 & 3 & 5 & $49(80.3 \%)$ \\
\hline Yes & 0 & 1 & 1 & 2 & 2 & 2 & 0 & 1 & 0 & 0 & 0 & 2 & 1 & 0 & 0 & 0 & $12(19.7 \%)$ \\
\hline \multicolumn{18}{|l|}{$\begin{array}{l}\text { What proportion of your } \\
\text { physicians/psychologists are treating } \\
\text { patients and their caregivers by } \\
\text { telephone? ( } n=60)\end{array}$} \\
\hline $0 \%$ & 0 & 0 & 0 & 0 & 0 & 0 & 0 & 0 & 0 & 0 & 0 & 0 & 0 & 0 & 1 & 0 & $1(1.6 \%)$ \\
\hline $0.1-4.9 \%$ & 0 & 0 & 0 & 0 & 0 & 1 & 0 & 0 & 1 & 0 & 0 & 0 & 0 & 0 & 0 & 0 & $2(3.3 \%)$ \\
\hline $5.0-9.9 \%$ & 0 & 0 & 1 & 0 & 0 & 1 & 0 & 0 & 0 & 0 & 0 & 0 & 0 & 0 & 0 & 0 & $2(3.3 \%)$ \\
\hline $10-24.9 \%$ & 1 & 0 & 0 & 0 & 1 & 5 & 0 & 1 & 0 & 1 & 0 & 0 & 0 & 1 & 0 & 2 & $12(20 \%)$ \\
\hline $25-49.9 \%$ & 0 & 0 & 0 & 1 & 1 & 2 & 2 & 2 & 0 & 0 & 0 & 0 & 0 & 0 & 0 & 0 & $8(13.3 \%)$ \\
\hline $50-74.9 \%$ & 0 & 1 & 0 & 1 & 1 & 1 & 0 & 1 & 1 & 1 & 2 & 0 & 0 & 2 & 0 & 1 & $12(20 \%)$ \\
\hline$>75 \%$ & 1 & 0 & 0 & 1 & 2 & 5 & 3 & 1 & 1 & 0 & 0 & 2 & 3 & 0 & 2 & 2 & $23(38.3 \%)$ \\
\hline \multicolumn{18}{|l|}{$\begin{array}{l}\text { What proportion of your } \\
\text { physicians/psychologists are treating } \\
\text { patients and their caregivers by } \\
\text { videoconference? }(n=61)\end{array}$} \\
\hline $0 \%$ & 0 & 0 & 0 & 0 & 0 & 2 & 0 & 0 & 0 & 0 & 0 & 0 & 0 & 0 & 1 & 0 & $3(4.9 \%)$ \\
\hline $0.1-4.9 \%$ & 1 & 0 & 0 & 0 & 1 & 2 & 1 & 0 & 2 & 0 & 0 & 1 & 1 & 1 & 1 & 0 & $11(18 \%)$ \\
\hline $5.0-9.9 \%$ & 0 & 0 & 1 & 0 & 0 & 1 & 0 & 2 & 0 & 0 & 1 & 0 & 0 & 0 & 0 & 0 & $5(8.2 \%)$ \\
\hline $10-24.9 \%$ & 0 & 0 & 0 & 2 & 1 & 3 & 0 & 1 & 0 & 1 & 1 & 0 & 1 & 0 & 0 & 2 & $12(19.7 \%)$ \\
\hline $25-49.9 \%$ & 1 & 0 & 0 & 0 & 1 & 6 & 2 & 1 & 0 & 0 & 0 & 0 & 0 & 0 & 0 & 2 & $13(21.3 \%)$ \\
\hline $50-74.9 \%$ & 0 & 1 & 0 & 1 & 0 & 1 & 1 & 0 & 1 & 0 & 0 & 1 & 0 & 1 & 1 & 1 & $9(14.8 \%)$ \\
\hline$>75 \%$ & 0 & 0 & 0 & 1 & 2 & 1 & 1 & 1 & 0 & 1 & 0 & 0 & 0 & 1 & 0 & 0 & $8(13.1 \%)$ \\
\hline \multicolumn{18}{|l|}{$\begin{array}{l}\text { Have you established a specific } \\
\text { procedure (e.g. videoconferences) to } \\
\text { support bereaved children to deal } \\
\text { with COVID-19-related grief or } \\
\text { trauma? }(n=61)\end{array}$} \\
\hline (1) on & 2 & 0 & 0 & 3 & 2 & 13 & 4 & 5 & 3 & 2 & 2 & 2 & 3 & 3 & 2 & 5 & $51(83.6 \%)$ \\
\hline Yes & 0 & 1 & 1 & 0 & 3 & 3 & 1 & 0 & 0 & 0 & 0 & 0 & 0 & 0 & 1 & 0 & $10(16.4 \%)$ \\
\hline \multicolumn{18}{|l|}{$\begin{array}{l}\text { What are your current major concerns } \\
\text { related to the pandemic with regard to } \\
\text { service provisions? }(n=58)\end{array}$} \\
\hline $\begin{array}{l}\text { We are not sufficiently in contact with } \\
\text { patients and families in need }\end{array}$ & 1 & 1 & 0 & 2 & 2 & 12 & 5 & 4 & 2 & 1 & 2 & 2 & 2 & 2 & 0 & 5 & $43(74.1 \%)$ \\
\hline $\begin{array}{r}\text { We are not able to maintain specific } \\
\text { therapeutic groups for distinct groups } \\
\text { of patients }\end{array}$ & 2 & 0 & 1 & 3 & 4 & 11 & 3 & 2 & 1 & 1 & 1 & 2 & 1 & 3 & 1 & 4 & $40(69 \%)$ \\
\hline $\begin{array}{l}\text { Members of my team are not as } \\
\text { attentive as usual to their patients }\end{array}$ & 0 & 0 & 0 & 0 & 0 & 2 & 1 & 1 & 0 & 0 & 0 & 0 & 0 & 1 & 0 & 0 & $5(8.6 \%)$ \\
\hline $\begin{array}{l}\text { Meetings to manage the crisis prevent } \\
\text { me from doing my work }\end{array}$ & 0 & 0 & 0 & 0 & 0 & 10 & 0 & 1 & 1 & 1 & 0 & 0 & 1 & 3 & 0 & 1 & $18(31 \%)$ \\
\hline $\begin{array}{l}\text { We are running a deficit because we } \\
\text { treat too few patients }\end{array}$ & 2 & 0 & 1 & 1 & 1 & 6 & 1 & 3 & 1 & 1 & 2 & 0 & 1 & 2 & 1 & 4 & $27(46.6 \%)$ \\
\hline Other & 0 & 0 & 0 & 0 & 1 & 1 & 0 & 1 & 0 & 0 & 1 & 1 & 0 & 0 & 1 & 0 & $6(10.3 \%)$ \\
\hline \multicolumn{18}{|c|}{ Perceived impact on patients and their psychopathology } \\
\hline $\begin{array}{l}\text { How do you perceive the impact of } \\
\text { COVID-19 pandemic on the mental } \\
\text { health of children and adolescents in } \\
\text { your region? }(n=64)\end{array}$ & & & & & & & & & & & & & & & & & \\
\hline Negligible & 0 & 0 & 0 & 0 & 0 & 0 & 0 & 0 & 0 & 0 & 0 & 0 & 0 & 0 & 0 & 0 & 0 \\
\hline
\end{tabular}


Table 2 (continued)

\begin{tabular}{|c|c|c|c|c|c|c|c|c|c|c|c|c|c|c|c|c|c|}
\hline Low & 0 & 0 & 0 & 0 & 0 & 3 & 3 & 2 & 0 & 1 & 0 & 0 & 1 & 0 & $\overline{0}$ & $\overline{0}$ & $10(15.6 \%)$ \\
\hline Medium & 2 & 1 & 1 & 3 & 1 & 8 & 2 & 2 & 1 & 1 & 1 & 1 & 2 & 2 & 3 & 2 & $33(51.6 \%)$ \\
\hline Strong & 0 & 0 & 0 & 0 & 4 & 5 & 1 & 2 & 2 & 0 & 1 & 1 & 0 & 1 & 1 & 3 & $21(32.8 \%)$ \\
\hline Extreme & 0 & 0 & 0 & 0 & 0 & 0 & 0 & 0 & 0 & 0 & 0 & 0 & 0 & 0 & 0 & 0 & 0 \\
\hline \multicolumn{18}{|l|}{$\begin{array}{l}\text { Has the COVID- } 19 \text { pandemic had an } \\
\text { influence on the contents of the } \\
\text { psychopathology your patients present } \\
\text { with? (e.g. fear of virus contamination } \\
\text { in OCD, anxiety, psychotic disorders) } \\
(n=63)\end{array}$} \\
\hline I am not aware of a single patient & 1 & 0 & 0 & 1 & 0 & 5 & 2 & 1 & 1 & 0 & 2 & 1 & 2 & 1 & 0 & 1 & $18(28.6 \%)$ \\
\hline$<5 \%$ & 1 & 0 & 0 & 1 & 4 & 8 & 3 & 2 & 0 & 0 & 0 & 1 & 0 & 2 & 0 & 2 & $24(38.1)$ \\
\hline $5.0-9.9 \%$ & 0 & 0 & 1 & 0 & 0 & 2 & 1 & 1 & 1 & 1 & 0 & 0 & 0 & 0 & 1 & 1 & $9(14.3 \%)$ \\
\hline $10-25 \%$ & 0 & 1 & 0 & 0 & 1 & 1 & 0 & 2 & 1 & 0 & 0 & 0 & 0 & 0 & 1 & 1 & $8(12.7 \%)$ \\
\hline$>25 \%$ & 0 & 0 & 0 & 1 & 0 & 0 & 0 & 0 & 0 & 0 & 0 & 0 & 1 & 0 & 2 & 0 & $4(6.3 \%)$ \\
\hline \multicolumn{18}{|l|}{$\begin{array}{l}\text { Are you seeing changes in the number } \\
\text { of referrals or requests for } \\
\text { assessments? ( } n=64)\end{array}$} \\
\hline No & 0 & 1 & 1 & 0 & 0 & 8 & 2 & 2 & 0 & 1 & 0 & 0 & 1 & 0 & 0 & 1 & $17(26.6 \%)$ \\
\hline Yes, a decline & 2 & 0 & 0 & 3 & 5 & 6 & 3 & 3 & 2 & 1 & 2 & 1 & 1 & 3 & 3 & 4 & $39(60.9 \%)$ \\
\hline Yes, an increase & 0 & 0 & 0 & 0 & 0 & 2 & 1 & 1 & 1 & 0 & 0 & 1 & 1 & 0 & 1 & 0 & $8(12.5 \%)$ \\
\hline \multicolumn{18}{|c|}{ 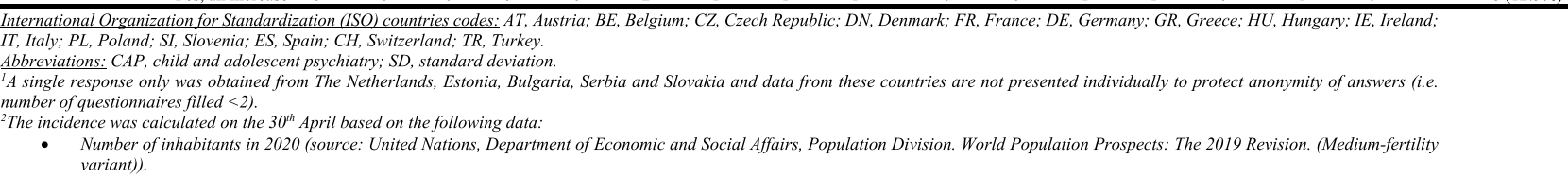 } \\
\hline
\end{tabular}

International Organization for Standardization (ISO) countries codes: $A T$ Austria, $B E$ Belgium, $C Z$ Czech Republic, $D K$ Denmark, $F R$ France, $D E$ Germany, GR Greece, HU Hungary, IE Ireland, IT Italy, PL Poland, SI Slovenia, ES Spain, CH Switzerland, TR Turkey

$C A P$ child and adolescent psychiatry, $S D$ standard deviation

${ }^{a}$ A single response only was obtained from The Netherlands, Estonia, Bulgaria, Serbia, and Slovakia and data from these countries are not presented individually to protect anonymity of answers (i.e. number of questionnaires filled $<2$ )

${ }^{\mathrm{b}}$ The incidence was calculated on the 30th April based on the following data: Number of inhabitants in 2020 (source: United Nations, Department of Economic and Social Affairs, Population Division. World Population Prospects: The 2019 Revision. (Medium-fertility variant))

Number of COVID-19 cases (source: COVID-19 Data Repository by the Center for Systems Science and Engineering (CSSE) at Johns Hopkins University)

reported that team members had tested positive for COVID19 but for the majority of them, it represented less than $1 \%$ of their team.

\section{Screening for COVID-19 in CAP services}

Prior to any hospital admission, approximately half of the CAP services only screened patients and family/household members for signs of infection, but 18/61 (30\%) systematically required a negative COVID-19 test. A majority of services $(n=37 / 61 ; 61 \%)$ were able to obtain a COVID-19 test within $24 \mathrm{~h}$.

\section{The reaction to COVID-19 at the organizational level and the effect on service provision}

The perceived mental condition of CAP teams to handle the crisis was considered as good (mean score $=62.6$ on a scale 0-100; $\mathrm{SD}=19.3$ ). The main interventions (endorsed by $>59 \%$ of respondents) used to reduce the stress of team members were allowing changes in the work schedule, encouraging home office, and allowing parents to stay at home to take care of their children. Similarly, respondents considered that their infrastructure was reasonably well equipped to handle COVID-19 patients (mean score $=56.6$ on a scale $0-100 ; \mathrm{SD}=25.4)$ despite the fact that most services were lacking personal protective equipment $(n=34 / 58$; $59 \%$ reported being only "partially" equipped). Telemedicine was used as a substitute for personal contacts with patients by an overwhelming majority of teams $(n=57 / 60$; $95 \%$ ), mostly by telephone (i.e. $58 \%$ of respondents had a majority of their physicians /psychologists treating patients over the phone vs. $28 \%$ for videoconferences) despite the fact that telemedicine was used sparsely before the pandemic $(n=12 / 61 ; 20 \%)$. Only 10/61 (16\%) respondents had established a specific procedure to support bereaved children to deal with COVID-19-related grief or trauma. Over $50 \%$ of respondents indicated that a COVID-19 hotline had been established for patients and their families.

A clear majority of respondents reported a major or extreme impact of COVID-19 on service provision (combined $n=47 / 59 ; 80 \%$ ), with restricted access to outpatient units $(n=50 / 56 ; 89 \%)$ and closure of daycare $(n=38 / 56$; $68 \%)$ and inpatient $(n=18 / 56 ; 32 \%)$ units. Thirty-five respondents out of $59(59 \%)$ reported that parts of their 
service had been closed or transformed for emergency COVID-19 use (not limited to CAP patients).

The vast majority $(n=21 / 24 ; 88 \%)$ of CAP services reported a substantial decrease in the average daily number of outpatients and beds occupied. Overall, the reduction was roughly two-thirds for outpatients $(69.9 \pm 135.3$ in 2019 to $24.7 \pm 35.2$ during COVID-19) and one-third for inpatients $(31.9 \pm 22$ versus $18.1 \pm 16.6)$. Major concerns affirmed by $>30 \%$ of respondents with regards to service provisions were: i) insufficient contact with patients and their families; ii) not being able to maintain specific therapeutic groups; iii) running a deficit because of treating too few patients, and iv) being prevented from doing clinical work due to more meetings to manage the crisis. Reassuringly, only five respondents $(8.5 \%)$ perceived their team members as less attentive to their patients than usual. Moderate or severe restrictions were imposed on contacts between patients and caregivers in $77 \%$ of the departments (44/52).

\section{Perceived impact on patients and their psychopathology}

Roughly half of the respondents observed a medium impact on their patients' mental health. At a national level and upon consideration of only countries providing four or more responses, the impact of the crisis on mental health seemed to be more related to the infection rate than to the severity of lockdown measures or the duration of school closure. French respondents, for instance, mainly reported a strong impact $(n=4 / 5$; $80 \%$ ); France experienced a strict lockdown and reported a high incidence of infections (256.3/100,000 on April 30, 2020). In contrast, Greece which also experienced an early and strict lockdown but succeeded in maintaining a low rate of infection, ten times lower than in France (24.9/100,000 on April 30, 2020), reported mainly a low impact of the COVID19 pandemic on pediatric mental health. Results were rather divergent in Germany (incidence 194.6/100,000; April 30), with a majority of respondents reporting a medium impact $(n=8 / 16 ; 50 \%)$, five (31\%) a strong, and three (19\%) a low impact, potentially reflecting regional differences in infection rates.

While respondents reported an increase in the amount of cases of anxiety disorders (36\%), conduct disorders (27\%), adjustment disorders (24\%), obsessive-compulsive disorders (24\%), and suicidal crises (24\%), 32\% of them highlighted no change (Fig. 1). Most respondents estimated that the COVID-19 pandemic had either no influence on the contents of their patients' psychopathology $(n=18 / 63 ; 29 \%)$ or in less than 5\% of their patients $(n=24 / 63: 38 \%)$.

\section{Analysis of free-text comments}

The thematic analysis of the free comments section of the questionnaire provided by a total of 19 respondents revealed that the reinforcement of inequalities is a concern mentioned by many heads of CAP services. Five respondents underlined the fact that confining families to their homes, putting financial pressure on parents, restricting access to school and/or childcare, and limiting access to child and adolescent mental health care, will inevitably put the most vulnerable kids at higher risks for developing mental health disorders. Two respondents also highlighted that the necessary reorganization of hospital services in order to be able to accommodate COVID-19 patients or closures of wards as a safety precaution decreased the number of beds available for CAP and consequently restricted access to inpatient care for adolescents. One participant drew attention to the fact that both reduced activity of youth welfare services and limited options for placements (foster care or daycare programs) during the crisis increased the length of hospital stays, thus further limiting the number of beds available in CAP services. Daycare units, if not closed, are at an elevated risk of contamination due to the more frequent contact of patients with the external environment. Accordingly, one respondent reported that the only two COVID-19-positive patients in their service were being cared for in a day unit.

Despite the adverse effects of the crisis, opportunities were also pointed out. Three respondents mentioned the rapid adoption of telepsychiatry to overcome the restriction of social distancing and to ensure or maintain contact with their patients. Two respondents pointed out that the decrease in clinical activity represented an opportunity to devote more time to research. In general, the crisis was seen as a unique occasion to rethink the way CAP services work and how services are organized ( $n=3$ ), despite the respondents' awareness of the overall unfavorable context for patients. Two respondents were concerned that the COVID-19 implications for children were not viewed as being a priority for politicians. One respondent expressed the concern that the lockdown potentially poses an infringement on the rights of children in light of restricted or suspended access to education and mental health care.

\section{Discussion}

This is the first survey on the impact of COVID-19 on CAP services at the beginning of the pandemic in Europe. From mid-April to mid-May 2020, 82 heads of CAP services in 20 countries responded anonymously to an online questionnaire prepared and diffused by ESCAP and its Research Academy. Notwithstanding highly variable incidence rates of COVID-19 infections between countries, the analysis revealed broadly comparable situations regarding lockdown 


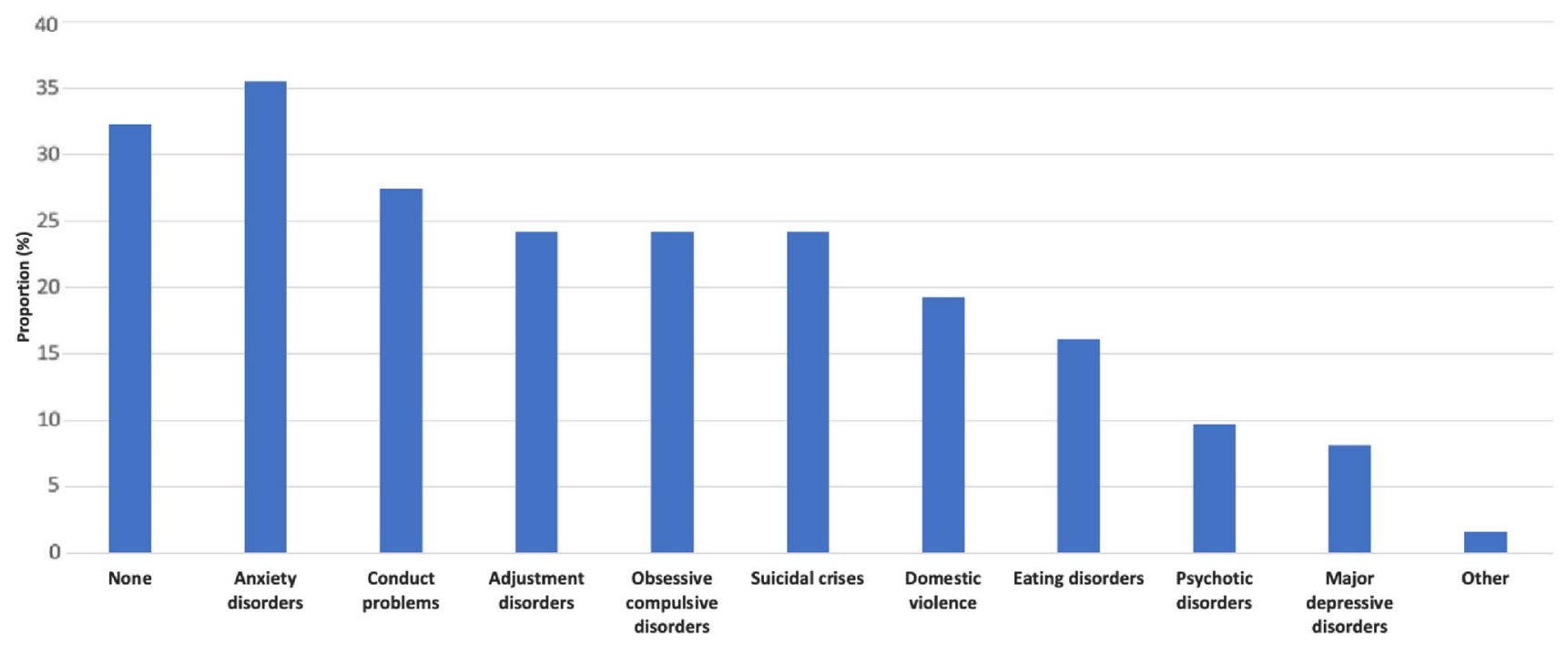

Fig. 1 Disorders reported by heads of child and adolescent services as showing an increase in the amount of cases (Compared with before the pandemic, is there a percentage increase in the amount of cases you and your team are seeing for the below disorders? $n=59$ )

measures, school closures, and reduced access to mental health care. Despite major reorganizations of services and the lack of preparation and resources, respondents had a rather positive perception of the capacity of their services to handle the crisis. Telepsychiatry was broadly adopted to provide the continuity of care in the context of the pandemic.

Previous studies have highlighted that public health emergencies can cause mental health problems, such as Wenchuan and Lushan earthquakes [8], 2014 Ebola outbreak [9], and SARS [10]. A recent study performed in an adult UK population found that adjusting for previous long-term trends, demographic, socioeconomic, and health-related factors, by late April 2020 mental health in the UK had deteriorated compared with pre-COVID-19 trends, more markedly in younger age groups [11]. Our results concerning the impact of the COVID-19 crisis on patients and their psychopathology were mixed. While an increase in anxiety disorders, conducts disorders and suicidal crises were observed, a third of respondents highlighted no change. The impact of the COVID-19 on the content of the psychopathology (e.g. fear of contamination by the virus in anxiety disorders) was also perceived as limited (i.e. no case or $<5 \%$ of cases) by two-thirds of respondents. However, a major concern of respondents was related to a possible delayed impact of the crisis due to the reduction of service provision. With this regard, some authors have warned about a possible psychiatric second wave due to the COVID-19 pandemic [12].

Interestingly, it seems that the COVID-19 crisis is acting as a catalyst, accelerating the adoption of new technologies, including telepsychiatry. In a recent editorial, Deschamps and colleagues highlighted the current needs for the development of CAP as a specialty and the training of future child and adolescent psychiatrists [13]. They insisted on the necessity for training to make use of the digital revolution, a topic that has rightly been addressed during the last meeting of the ESCAP Research Academy [14]. Beyond these general observations, the application of digital medicine should be specifically investigated in the context of the COVID-19 crisis, which will certainly have a long-lasting and wide-ranging effect on the field of CAP, not only on mental health care delivery and configurations but also on patient experience and expectations [15-17]. A recent ESCAP editorial [18] provides clinicians with basic guidance deemed important in light of the perceived lack of regulation.

This study is not without limitations. The overall response rate was below $50 \%$ and despite a reasonable number of questionnaires filled in at the European level, less than five questionnaires were received for most countries. Hence, results at the national level should be interpreted with caution, especially since for a given country, the lockdown and school closure measures, as well as the incidence of COVID19 cases, can also differ greatly between regions. Due to the limited time available to launch the survey, contact emails were missing for heads of CAP services in eleven ESCAP member countries. We will endeavor to include these countries in the next two parts of the survey. Last but not least, most questions were subject to personal interpretation and some answers also required rough estimations. Therefore, it is important to keep in mind that the collected data represents general impressions from heads of services.

Despite these limitations and substantial national differences in COVID-19 infection rates during April and May of 2020, overall these results suggest a rather astonishingly similar situation in Europe. Given the novelty of this crisis 
and the uncertainty of its evolution in the following months, future data from the CovCAP survey will be important to provide more information on the impact of COVID-19 on CAP services (e.g. lasting effects of the reorganization of services, increased demand as school referrals restart, etc.) and on children and adolescents' mental health.

Supplementary Information The online version contains supplementary material available at https://doi.org/10.1007/s00787-020-01699-x.

Acknowledgments The authors would like to thank the members of the ESCAP Board who gave their precious feedback on the first version of the questionnaire. They also would like to thank all the respondents to this study who took the time to complete the questionnaire. PK is supported by a Fellowship from the Adrian and Simone Frutiger Foundation. We thank the Center for Clinical Research at CHUV for the provision of the REDCap ${ }^{\circledR}$ platform and their logistical support. COVID-19 Child and Adolescent Psychiatry Consortium: Tobias Banaschewski: Department of Child and Adolescent Psychiatry and Psychotherapy, Central Institute of Mental Health, Medical Faculty Mannheim, Heidelberg University, J5, Mannheim 68159, Germany, Stephan Bender: University Hospital Cologne and Medical Faculty, University of Cologne, Germany, Györgyi Csábi: University of Pécs, Medical School, Department of Paediatrics, Hungary, Füsun Çuhadaroğlu: Hacettepe University, Ankara, Turkey, Elona Dashi: Child and Adolescent Psychiatric Triane, University Medical Center "Mother Theresa", Tirane, Albania, Richard Delorme: Child and Adolescent Psychiatry Department, Robert Debré Hospital, APHP, Paris, France, Maja Drobnic Radobuljac: University Psychiatric Hospital Ljubljana and Medical Faculty, University of Ljubljana, Slovenia, Stephan Eliez: University of Geneva School of Medicine, Department of Psychiatry, Geneva, Switzerland, Mette Falkenberg Krantz: CORE- Copenhagen Research Centre for Mental Health, Mental Health Center Copenhagen, Capital Region of Denmark, Copenhagen University Hospital, Gentofte Hospitalsvej 15, opg. 15, 1. sal., 2900 Hellerup, Denmark, Oliver Fricke: Gemeinschaftskrankenhaus Herdecke and Witten/Herdecke University, Germany, Miriam Gerstenberg: Psychiatric University Hospital Zurich, Department of Child and Adolescent Psychiatry and Psychotherapy, Zurich, Switzerland, Ioanna Giannopoulou: National and Kapodistrian University of Athens, School of Medicine, 2nd Department of Psychiatry, Attikon University General Hospital, Athens, Greece, Montserrat Graell: Hospital Infantil Universitario Niño Jesus, Universidad Autónoma de Madrid, Cibersam, Madrid, Spain, Hojka Gregoric Kumperscak: University Medical Centre Maribor and Faculty of medicine Maribor, Maribor, Slovenia, Beate Herpertz-Dahlmann: Department of Child and Adolescent Psychiatry, Psychosomatics and Psychotherapy, Technical University RWTH, Aachen, Germany, Mercedes Huscsava: Medical University of Vienna, Vienna, Austria, Michael Kaess: University Hospital of Child and Adolescent Psychiatry and Psychotherapy, University of Bern, Switzerland, Krisztina Kapornai: University of Szeged, Department of Pediatrics, Child and Adolescent Psychiatric Unit, Hungary, Andreas Karwautz: Child and Adolescent Psychiatry, Medical University Vienna, Austria, Dominika Kresakova: Service of Child and Adolescent Psychiatry, Department of Psychiatry, Lausanne University Hospital, Lausanne, Switzerland, Michael Kölch: Dept. of Child and Adolescent Psychiatry, Neurology, Psychosomatics and Psychotherapy, Rostock University Medical Center, Germany, Konstantinos Kotsis: Assistant Professor of Child and Adolescent Psychiatry, Department of Psychiatry, Faculty of Medicine, School of Health Sciences, University of Ioannina, Ioannina, Greece, Luisa Lazaro: Department of Child and Adolescent Psychiatry and Psychology, IDIBAPS, CIBERSAM, University of Barcelona, Spain, Eva Moehler: Saarland University Hospitality, SaarBrücken, Germany, M. Goretti Morón-Nozaleda: Child and Adolescent Psychiatry and Clinical Psychology Department - Hospital Infantil Universitario Niño Jesús, Madrid, Spain, Gonca Özyurt: Katip çelebi university medical school department of child psychiatry, Izmir, Turkey, Bea Pászthy: Semmelweis University, 1st Department of Pediatrics, Budapest, Hungary, Jiri Podlipny: Department of Psychiatry, University Hospital in Plzen, Faculty of Medicine in Plzen, Charles University, Czech Republic, Diane Purper-Ouakil: CHU Montpellier, MPEA Child and Adolescent Psychiatry Saint Eloi Hospital, France, Barbara Remberk: Child and Adolescent Psychiatry Department, Institute of Psychiatry and Neurology, Warsaw, Poland, Aspasia Serdari: Department of Child and Adolescent Psychiatry, Democritus University of Thrace, Greece, Lise Eilin Stene: Norwegian Centre for Violence and Traumatic Stress Studies (NKVTS), Oslo, Norway, Leonhard Thun-Hohenstein: Paracelsus Medical Private University \& Salzburger Landeskliniken, Salzburg, Austria, Jana Trebaticka: Department of Paediatric Psychiatry, Faculty of Medicine Comenius University, Bratislava, The National Institute of Children's Diseases, Slovakia, Dirk van West: ZNA-UKJA, University of Antwerp (UA), University of Brussels (VUB), Belgium, Benedetto Vitiello: University of Turin, Turin, Italy, Héloïse Young: Centre Hospitalier de Versailles, le Chesnay, France - Inserm « psychiatrie du développement » UMR 1018, France, Nihal Yurteri: Department of Child and Adolescent Psychiatry, Faculty of Medicine, Düzce University, Düzce, Turkey, Florian Daniel Zepf: Department of Child and Adolescent Psychiatry, Psychosomatic Medicine and Psychotherapy, Jena University Hospital, Friedrich Schiller University Jena, Jena, Germany, Anna Zielinska-Wieniawska: Department of Child and Adolescent Psychiatry, Medical University of Warsaw, Warsaw, Poland, Alessandro Zuddas: Department of Biomedical Science and "G. Brotzu" Hospital Trust, Child and Adolescent Neuropsychiatry, University of Cagliari, Cagliari, Italy.

Auhtor contributions AR, PK, LK, and JH designed the study and wrote the protocol. JH, AR, and PK designed the first version of the questionnaire, which was then corrected and improved by LK, DA, and members of the Board of the European Society for Child and Adolescent Psychiatry (ESCAP). AR and PK conducted the analyses and produced the first draft of the manuscript. JH, LK, and DA contributed to the interpretation of the data, drafted portions and substantively revised all drafts of the manuscript for important intellectual content. All authors contributed to and have approved the final manuscript. AR and PK take responsibility for the integrity of the data and the accuracy of the data analysis.

\section{Compliance with ethical standards}

Conflict of interest The authors have no conflict of interest to declare related to this study.

Ethical approval Ethics committee approval was not required for this anonymous web-based questionnaire study.

\section{References}

1. Moreno C, Wykes T, Galderisi S et al (2020) How mental health care should change as a consequence of the COVID-19 pandemic. Lancet Psychiatry. https://doi.org/10.1016/S2215-0366(20) 30307-2

2. Golberstein E, Wen H, Miller BF (2020) Coronavirus disease 2019 (COVID-19) and Mental Health for Children and Adolescents. JAMA Pediatr. https://doi.org/10.1001/jamapediatrics.2020.1456

3. Brooks SK, Webster RK, Smith LE et al (2020) The psychological impact of quarantine and how to reduce it: rapid review of 
the evidence. Lancet Lond Engl 395:912-920. https://doi.org/10. 1016/S0140-6736(20)30460-8

4. Golberstein E, Gonzales G, Meara E (2019) How do economic downturns affect the mental health of children? Evidence from the National Health Interview Survey. Health Econ 28:955-970. https://doi.org/10.1002/hec.3885

5. Revet A, Hebebrand J, Klauser P (2017) The 2017 ESCAP Research Academy Workshop: bright perspectives for child and adolescent psychiatry. Eur Child Adolesc Psychiatry. https://doi. org/10.1007/s00787-017-1036-5

6. Jones J, Hunter D (1995) Consensus methods for medical and health services research. BMJ 311:376-380. https://doi.org/10. 1136/bmj.311.7001.376

7. Castleberry A, Nolen A (2018) Thematic analysis of qualitative research data: is it as easy as it sounds? Curr Pharm Teach Learn 10:807-815. https://doi.org/10.1016/j.cptl.2018.03.019

8. Xie Z, Xu J, Wu Z (2017) Mental health problems among survivors in hard-hit areas of the 5.12 Wenchuan and 4.20 Lushan earthquakes. J Ment Health Abingdon Engl 26:43-49. https://doi. org/10.1080/09638237.2016.1276525

9. Shultz JM, Baingana F, Neria Y (2015) The 2014 Ebola outbreak and mental health: current status and recommended response. JAMA 313:567-568. https://doi.org/10.1001/jama.2014.17934

10. Mak IWC, Chu CM, Pan PC et al (2009) Long-term psychiatric morbidities among SARS survivors. Gen Hosp Psychiatry 31:318-326. https://doi.org/10.1016/j.genhosppsych.2009.03.001

11. Pierce M, Hope H, Ford T et al (2020) Mental health before and during the COVID-19 pandemic: a longitudinal probability sample survey of the UK population. Lancet Psychiatry. https://doi. org/10.1016/S2215-0366(20)30308-4
12. Stefana A, Youngstrom EA, Hopwood CJ, Dakanalis A (2020) The COVID-19 pandemic brings a second wave of social isolation and disrupted services. Eur Arch Psychiatry Clin Neurosci. https:// doi.org/10.1007/s00406-020-01137-8

13. Deschamps P, Hebebrand J, Jacobs B et al (2020) Training for child and adolescent psychiatry in the twenty-first century. Eur Child Adolesc Psychiatry. https://doi.org/10.1007/ s00787-019-01467-6

14. Klauser P, Hebebrand J, Kehoe LA et al (2019) The 2019 ESCAP research academy workshop: how novel technologies are impacting child and adolescent psychiatry. Eur Child Adolesc Psychiatry. https://doi.org/10.1007/s00787-019-01378-6

15. Holmes EA, O'Connor RC, Perry VH et al (2020) Multidisciplinary research priorities for the COVID-19 pandemic: a call for action for mental health science. Lancet Psychiatry 7:547-560. https://doi.org/10.1016/S2215-0366(20)30168-1

16. Shore JH, Schneck CD, Mishkind MC (2020) Telepsychiatry and the Coronavirus Disease 2019 Pandemic-current and future outcomes of the rapid virtualization of psychiatric care. JAMA Psychiatry. https://doi.org/10.1001/jamapsychiatry.2020.1643

17. Kalin ML, Garlow SJ, Thertus K, Peterson MJ (2020) Rapid implementation of telehealth in hospital psychiatry in response to COVID-19. Am J Psychiatry 177:636-637. https://doi.org/10. 1176/appi.ajp.2020.20040372

18. Allgaier K, Schmid J, Hollmann K et al (2020) Times are changing: digitalisation in child and adolescent psychotherapy. Eur Child Adolesc Psychiatry. https://doi.org/10.1007/ s00787-020-01610-8

\section{Authors and Affiliations}

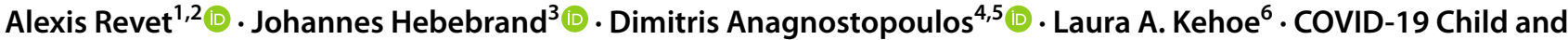 Adolescent Psychiatry Consortium • Paul Klauser ${ }^{7,8}$ (B)}

\author{
Johannes Hebebrand \\ johannes.hebebrand@uni-due.de \\ Dimitris Anagnostopoulos \\ dimitris1952@gmail.com \\ Laura A. Kehoe \\ laura.a.kehoe@gmail.com \\ Paul Klauser \\ paulklauser@escap.eu
}

$1 \quad$ Service Universitaire de Psychiatrie de L'Enfant et de L'Adolescent, CHU de Toulouse, Hôpital Purpan, Place du Dr Baylac, TSA 40031, 31059 Toulouse cedex 9, France

2 UMR 1027, Inserm, Université Toulouse III, Toulouse, France

3 Department of Child and Adolescent Psychiatry, Psychosomatics and Psychotherapy, LVR Klinikum Essen, University Hospital Essen, University of Duisburg-Essen, Essen, Germany
4 Medical School, National and Kapodistrian University of Athens, Athens, Greece

5 President of European Society for Child Adolescent Psychiatry, National and Kapodistrian University of Athens, Athens, Greece

6 Medical Communications, Avenue des Cadolles 12D, 2000 Neuchatel, Switzerland

7 Service of Child and Adolescent Psychiatry, Department of Psychiatry, Lausanne University Hospital, Lausanne, Switzerland

8 Center for Psychiatric Neuroscience, Department of Psychiatry, Lausanne University Hospital, Lausanne, Switzerland 